\title{
Les suppliants et leurs rameaux au début de l'CEdipe-roi
} Marie Delcourt

Citer ce document / Cite this document :

Delcourt Marie. Les suppliants et leurs rameaux au début de l'@Edipe-roi. In: L'antiquité classique, Tome 6, fasc. 1, 1937. pp. 63-70;

doi : 10.3406/antiq.1937.3040

http://www.persee.fr/doc/antiq_0770-2817_1937_num_6_1_3040

Document généré le 15/03/2016 


\title{
LES SUPPLIANTS ET LEURS RAMEAUX
}

\section{AU DÉBUT D’CEDIPE-ROI}

\author{
par Marie Delcourt.
}

Edipe, parlant aux suppliants qui sont devant sa maison, leur demande:

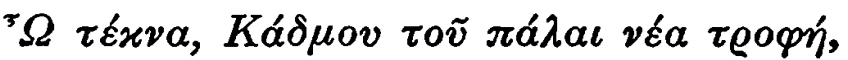

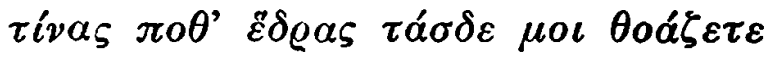

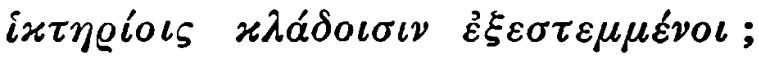

ce que le premier rhétoricien venu traduira par: "Mes enfants, derniers descendants de l'antique Cadmus, qu'est-ce que cette assemblée que vous tenez ici, couronnés de rameaux suppliants? "Il remarquera que les rameaux couronnent, non chaque suppliant, mais le groupe qu'Edipe voit d'un peu haut, les branches droites dépassant les tètes. La même nuance se dégage clairement de l'ex-

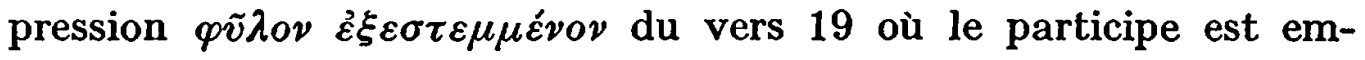
ployé sans instrumental. Les rameaux eux-mêmes sont mentionnés une dernière fois par CEdipe au moment où il invite le peuple à quitter le palais en emportant les branches

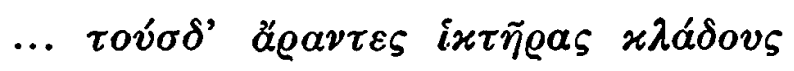

La traduction ci-dessus proposée pour le vers 3 paraît donc satisfaisante et corroborée par la suite du texte. On s'excuse de devoir consacrer plusieurs pages à la défendre.

C'est le commentaire du scholiaste qui a amené les critiques modernes à chercher un autre sens à ce vers parfaitement clair. Ils ne se sont pas aperçus que le scholion contient en réalité trois notes indépendantes, que la troisième s'est ajoutée aux deux autres à la suite d'une simple association d'idées et qu'elle ne doit pas entrer en ligne de compte pour l'explication du texte. Voici cette note, que je divise d'après ses articulations naturelles:

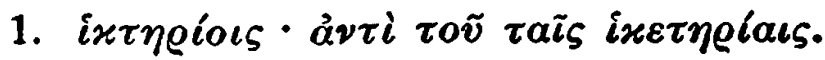




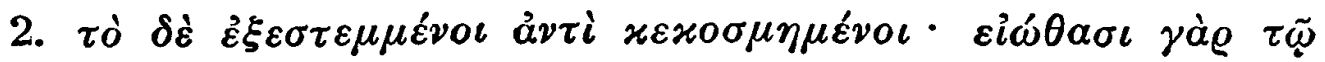

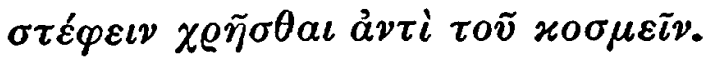

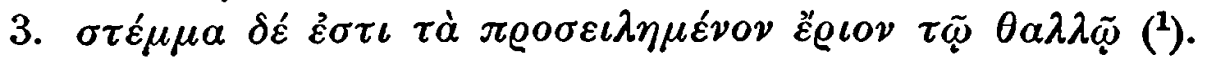

Il suffit de lire ces lignes pour voir que les nos 1 et 2 conduisent tout droit à l'interprétation toute simple: couronnés (parés, dit le scholiaste qu'une métaphore hardie effraie un peu) de rameaux suppliants. C'est l'explication relative à la bandelette qui a faussé l'exégèse à partir du moment où l'on a voulu la mettre en rapport direct avec le texte de Sophocle.

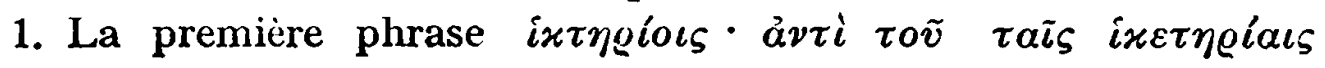

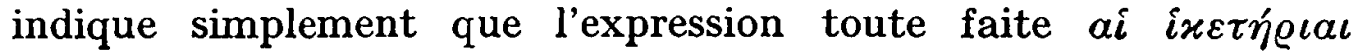

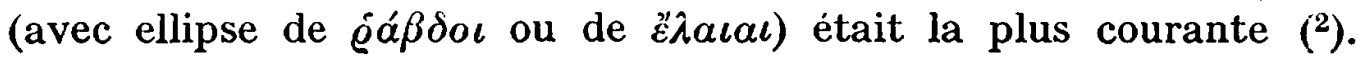

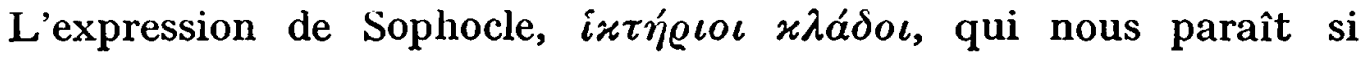
simple, était probablement poétique. Le scholiaste n'aurait pas pris la peine de gloser une expression complète par une expression elliptique si cette dernière n'avait pas été rendue familière par des formules rituelles.

2. S'il fallait prendre à la lettre la seconde partie du scholion,

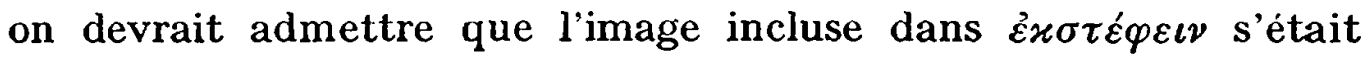
usée au point que le mot devint l'équivalent du terme général

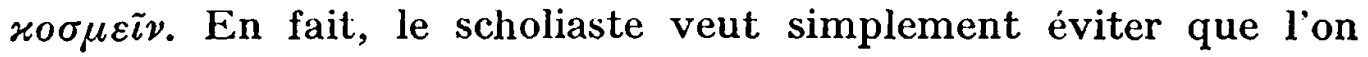
s'imagine les 'Thébains portant une couronne sur la tête, comme

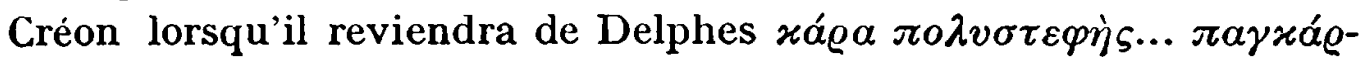

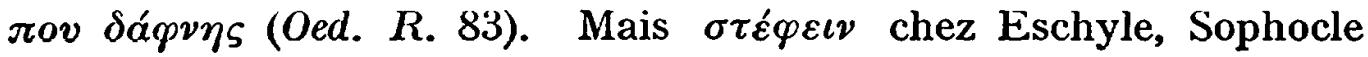

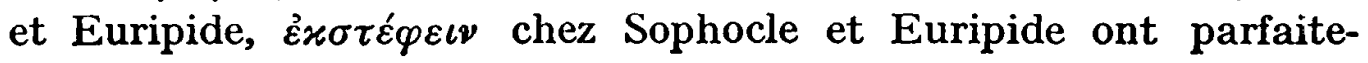
ment gardé leur valeur étymologique de entourer d'ornements. Des passages comme Eumén. $44\left(^{3}\right)$, comme Herc. Fur. 526, comme Alceste 170-172, montrent que le verbe, simple ou composé, avait gardé sa valeur descriptive, et qu'il désignait un ornement qui entoure, dépasse l'objet et attire l'attention.

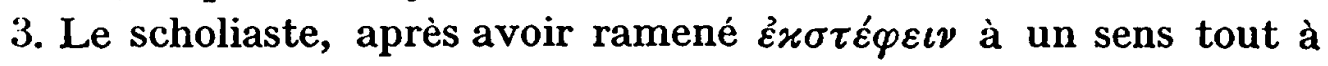
fait général, croit bon d'en suggérer une étymologie et il le rat-

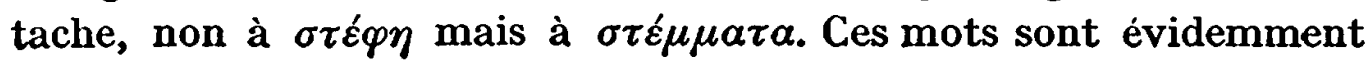

(1) Suidas, s.v. $\grave{\varepsilon} \xi \varepsilon \sigma \tau \varepsilon \mu \mu \dot{\varepsilon} v o$ don do le même texte que le schol. avec renvoi au vers de Sophocle.

(2) Elle figure chez Eschyle (Suppl. 192) et chez Euripide (Iph. Aul. 1216) Ni l'un ni l'autre, à notre connaissance, n'emploient la forme ixtýeıos.

(3) Vide infra, p. 67. 
de la mème famille, mais il me paraît évident que les $\sigma \tau \varepsilon \dot{\varepsilon} \mu \mu \alpha \tau \alpha$ ne jouent aucun rôle dans le vers de Sophocle. Et, malheureusement, les exégètes modernes ont voulu à tout prix introduire ces bandelettes dans l'ensemble d'images évoqué par le vers 3 . Et, depuis Hermann $\left(^{1}\right)$, au lieu de référer $\dot{\varepsilon} \xi \varepsilon \sigma \tau \varepsilon \mu \mu \varepsilon \dot{\varepsilon} v o \iota$ aux suppliants couronnés de rameaux, on le réfère aux rameaux entourés de ban-

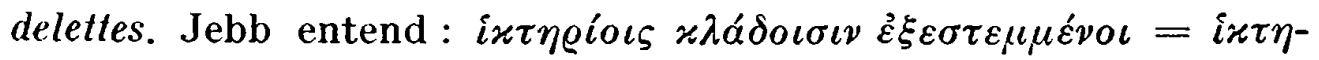

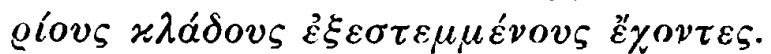

Evidemment, si Sophocle écrivait ainsi, on pourrait l'accuser de préciosité. Mais le vers peut-il avoir ce sens?

Jebb allègue deux passages de Xénophon:

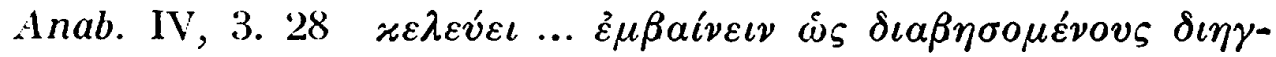

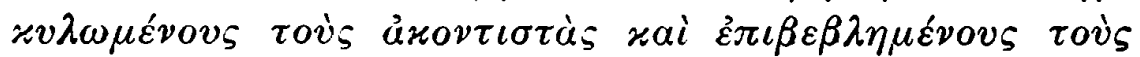

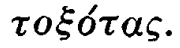

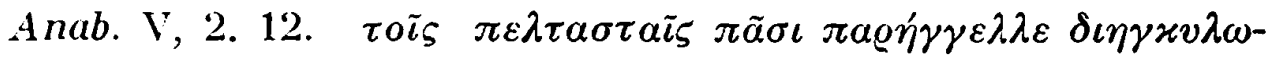

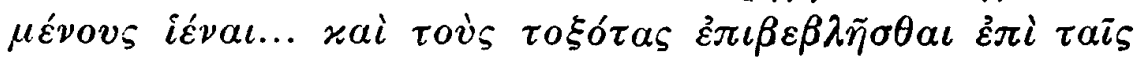

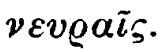

ce qui, d'après lui, semble revenir (car il donne bien une transcription anglaise, mais non une transcription grecque) à l'équation suivante :

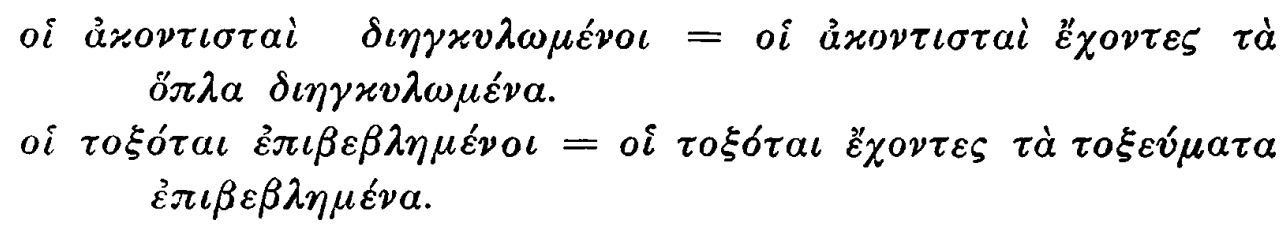

On le voit tout de suite : ces deux exemples ne sont même pas parallèles au vers de Sophocle (ici, Jebb suppose sous-entendus

(1) Voici la note de Hermann (1823), source de celle de Dindorf : $\xi \xi \varepsilon \sigma \tau$. Male interpres: coronati. Vertere poterat: instructi; non coronis erant redimiti, sed manibus jerebant ramos oleae lana obvolutos qui graecis stemmata vocantur, Sic Chryses Iliadis initio. Logique avec lui-même, Hermann ne donne aucune note pour le vers 19 où $\varepsilon \xi \varepsilon \sigma \tau \varepsilon \mu \mu \varepsilon$ v́vo, pris absolument, ne saurait se traduire par instructi. Masqueray traduit le début par : "... pourquoi donc êtes-vous ainsi à genoux, avec des rameaux suppliants couronnés de bandelettes? " et le vers 19 par : "le reste du peuple couronné de rameaux", de telle sorte que le mot rameaux n'apparait à l'instrumental, dans la traduction, que dans le vers où il ne figure pas en grec. De tels illogismes auraient dú attirer l'attention et faire déceler une erreur. 


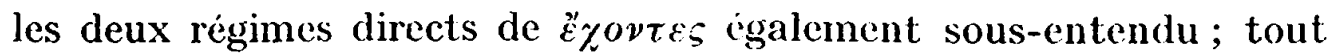
à l'heure, il le tirait d'un datif). Èt, de plus près on les regarde, mieux on se rend comple quils ne peuvent ètre invoqués pour l'interprétation du vers d'EAdipe. Xénophon rapporte des ordres, expressions toutes faites, qui, au discours direct, se composaient probablement d'un infinitif seul $\iota_{\iota} \gamma \% v \lambda \sigma \tilde{v} \sigma 0 \alpha \iota, \dot{\varepsilon} \pi \iota \beta \alpha \dot{\lambda}-$ $\lambda \varepsilon \sigma \theta a \iota$. Le premier de ces verbes est connu par Xénophon seul : le second est employé dans quantité de tournures elliptiques. Jebb veut que les deux formes employées soient passives. Tout donne à penser qu'elles sont moyennes $\left(^{1}\right)$. Au contraire, dans le passage de Sophocle, le participe précédé d'un datif devait ètre senti comme un passif et le participe comme un instrumental par tous les auditeurs comme par tous les lecteurs au cour simple.

L'interprétation : "couronnés de rameaux n est imposée par l'usage courant du grec. La note de Dindorf : "ramos dicit oleaginos lana obvolutos, qui $\sigma \tau \dot{\varepsilon} \mu \mu \alpha \tau \alpha$ et $\sigma \tau \dot{\varepsilon} \varphi \eta$ dicuntur, unde qui eos ma-

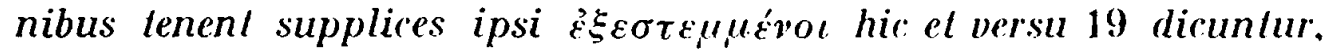

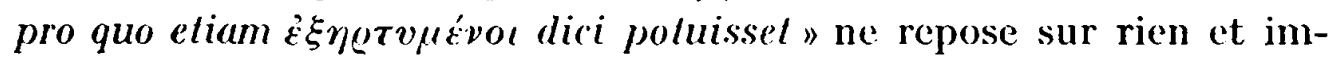
plique une contradiction, car, si $\bar{\xi} \xi \sigma \tau \varepsilon \mu \mu \varepsilon v^{\prime} \sigma s$ équivaut à $\sigma \tau \varepsilon \mu \mu \alpha \sigma \iota$

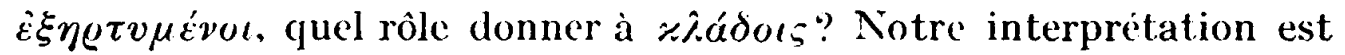
confirmée par limitation de Virgile qui se souvient certes de Sophocle lorsqu'il dit des ambassadeurs d'Enée (Inn. VII, 154) qu'ils sont ramis velati Palladis. Elle est enfin celle du scholiaste lui-même, qui, dans la troisième partie de sa note, explique $\sigma \tau \varepsilon \dot{\mu} \mu \mu \alpha \tau \alpha$ et non $\dot{\varepsilon} \xi \varepsilon \sigma \tau \varepsilon \mu \mu \varepsilon \dot{v} v \iota$. Ce n'est pas sa faute si des successeurs trop zélés ont incorporé de force, dans un vers parfaitement clair, un détail d'origine étrangère.

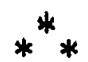

Si le scholiaste a parlé de bandelettes à propos de notre passage, c'est qu'il s'attendait que Sophocle les mentionnât. Elles figurent dans plusieurs scènes qui ont une ressemblance apparente avec la nôtre. Le silence même de Sophocle indique une intention qu'il peut être intéressant de préciser.

(1) Notamment l'emploi d'un infinitif moyen à sens actif dans le second exemple. Le verbe $\delta \iota \eta \gamma x v \lambda \varepsilon i \sigma \theta \alpha \iota$ qui existe à còté de $\delta \iota \eta \gamma x v \lambda \circ \tilde{v \sigma} \theta \alpha \iota$, s'emploie au moyen dans le sens actif de "tenir une arme prête à partir *. 
Les exégètes anciens et modernes ont présentes à l'esprit des scènes de supplication devenues classiques. Les Suppliantes d'E-

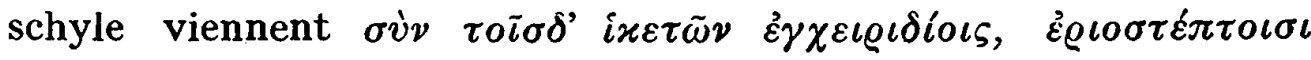
$\varkappa \lambda a ́ \delta o \iota \sigma \iota$, " avec cet attribut des bras suppliants, ces rameaux. ceints de laine" (trad. Mazon). Plus loin, on parle de leurs $\lambda \varepsilon v$ -

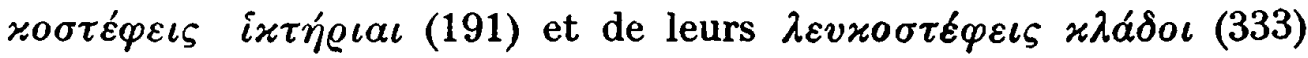
$\mathrm{Au}$ début des Euménides, la Pythie décrit Oreste

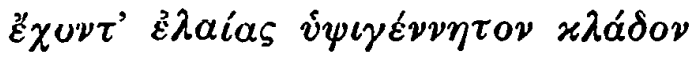

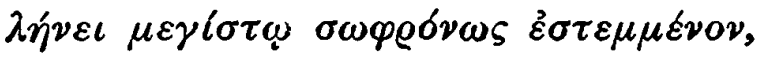

$$
\begin{aligned}
& \dot{\alpha} \varrho \gamma \tilde{\eta} \tau \iota \mu \alpha \lambda \lambda \tilde{\omega} \text {. }
\end{aligned}
$$

"tenant un long rameau d'olivier, dévotement entouré d'un épais réseau de bandelettes, une vraie toison blanche" (trad. Mazon)

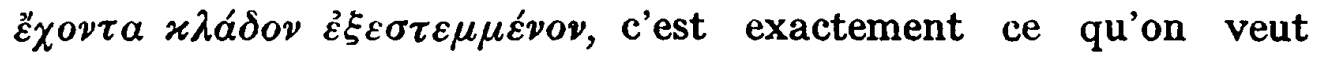
faire dire à Sophocle.

Dans les deux drames d'Eschyle, les bandelettes sont expressément mentionnées, et, dans le second avec une emphase voulue. On voit aisément pourquoi. Les Suppliantes soulignent, au cours de toute leur prière, que les rites ont été scrupuleusement observés, que les formes sont sauves. Oreste est dans le même cas : il a si peur de n'être pas en règle que son rameau d'olivier est plus grand qu'on ne les taille d'habitude et que les bandelettes $y$ font comme la toison d'une quenouille. Sophocle aussi connaissait ces deux scènes, mais, contrairement à ce que semblent croire ses commentateurs, il n'a pas voulu imiter Eschyle, mais bien faire autre chose que lui.

L'invocation au prologue d'Edipe-Roi repose sur une situation tout à fait différente de celle des Suppliantes et d'Oreste, sur une situation dont la nouveauté devait être vivement sentie par les spectateurs.

C'est à peine une prière rituelle. Elle s'adresse aux dieux du

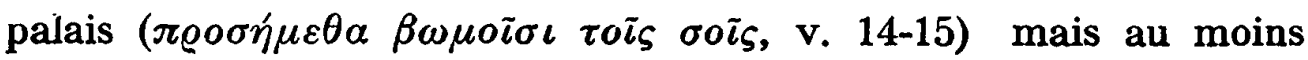
autant à Edipe lui-même. Ce devait être, pour les Grecs du ve s., un curieux retournement que de voir un prêtre venir en suppliant à la porte d'un roi. Du reste, le grand prêtre dit:

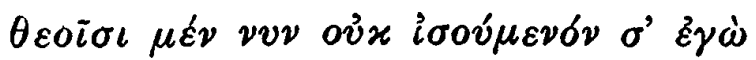

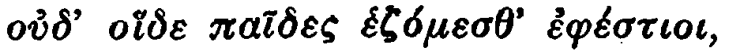

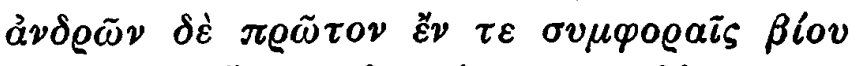

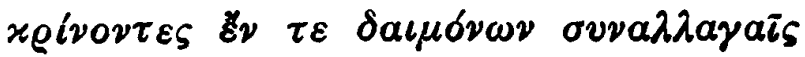


Sophocle n'a donc aucune raison d'insister comme Eschyle sur la stricte observance des rites. Il mentionne simplement les rameaux qui, levés en couronne autour de cetle foule, font un tableau frappant et, plus loin, donneront lieu à un jeu de scène, lorsqu'Edipe, comme Pélasgos, invite les suppliants à enlever les branches (CEd. R. 143, Suppl. 181). Qu'il y ait un parenté scénique entre le prologue d'(Edipe et la première partie des Suppliantes, c'est l'évidence mème. Mais Sophocle s'est plu à mettre dans une forme connue un contenu nouveau.

La scène de la supplication dans (Edipe-Roi est tout à fait unique dans la tragédie. Nulle part on ne trouve semblable contamination entre une prière religisuse et un conseil demande $a$ un roi. Il suffit de relire les deux pièces pour messurer la difference entre l'intervention de Pélasgos et celle d'CEdipe. P ílasgos se demande s'il doit accomplir, aux dépens de son peuple, la volonté de \%eus efficacement sollicité par les princesses : il est fait, malgré lui, linstrument de la justice et la force des rites l'oblige d'intervenir pour empecher une souillure. Identique est la position de Thésée, instrument de Déméter au bénéfice des Argiens dans les Suppliantes d'Euripide. I es Danaïdes, les mires argiennes dictent à un roi le message d'un dieu. Le roi, cruellement contraint, reconnaît le' caractère obligatoire du message. 1)ans (Edipe-Roi tout reste sur le plan humain. Le grand prètre, bien loin de dicter son devoir à Edipe, lui demande de trouver une solution que les dieux, jusquà présent, nont pas révélé. Et ces dieux du palais, sitòt mentionnés, retombent dans l'oubli. C'est d'Edipe que l'on espère quelque chose. Edipe est le seul homme que l'on juge capable de comprendre la mystérieuse volonté des dieux, d'entendre leur voix. Ironie tragique.

Aprés queledipe a reçu, de la bouche de Créon, la réponse de l'oracle qui doit mettre fin au malheur public, il invite les suppliants à emporter leurs rameaux. De ce passage et de deux autres des Suppliantes dEuripide, Jeb), dans son commentaire a vers 3 d'CEdipe-Roi, tire la règle suivante: "on enlevait les rameaux de l'autel lorsqu'on avait obtenu satisfaction, on les y laissait dans le cas conlraire". Est-ce exact"

Il faut tout d'abord écarter un des passages invoqués par Jebb. 
Lorsque Thésée (Suppl. 359) prie les mères de laisser passer Aethra "enfermée dans le cercle sacré des saintes bandelettes" (102-3)

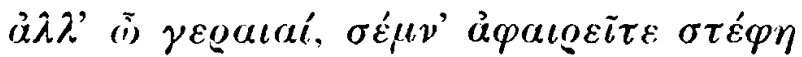

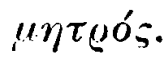

il décrit simplement un mouvement de scène sous grande signifi(ation religieuse.

I.es vers 258-262 sont plus intéressants. Adraste dit aux mères, après que Thésée a refusé de défendre leur cause :

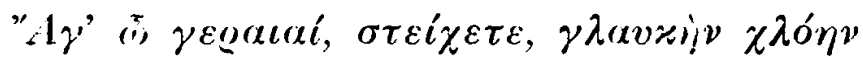

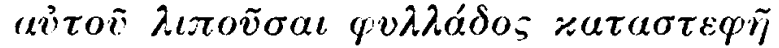

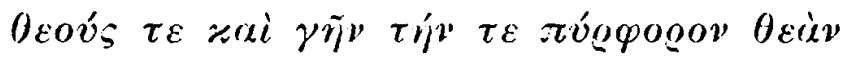

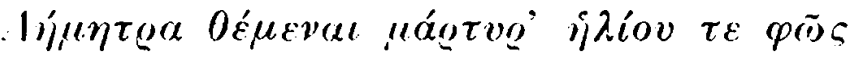

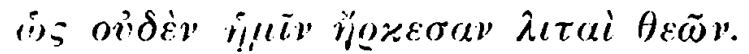

"Allons, vieilles, en marche, laissez cette claire verdure, laissezlà ces rameaux couronnés de feuillage, en prenant à témoin les dieux et la Terre, Déméter porte-flamme et l'éclat du Soleil que nous fî̀mes au Ciel d'inutiles prières" (trad. H. (irégoire).

Mais il est bien difficile de tirer une loi de deux exemples seulement, étant donné que nous n'avons aucun texte qui nous autorise formellement à généraliser. L'étude des jeux de scène dans les Suppliantes d'Eschyle semble indiquer qu'il n'y avait pas de rite obligatoire. Le roi commence par dire à Danaos d'aller porter les rameaux sur d'autres autels, afin d'émouvoir les Argiens (480185), puis il prie les jeunes filles de laisser là les rameaux, symboles de leur misère:

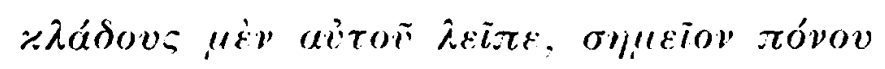

('est à dire qu'il continuera à less protéger mème si les rameaux rituels cessent leur effet magique. Elles hísitent du reste à se confier à lui. Le contraste entre le formalisme des Danaïdes et le sentiment de la justice pure che\% le roi se marque ici une fois de plus.

Peut-ètre peut-on tirer de ces passages la conclusion suivante: Le rameau n'a de force contraignante que s'il est tenu en main par le suppliant à côté de l'autel d'un dieu. Dès que l'on quitte l'autel, la supplication rituelle cesse, que l'on garde ou non les branches sur la saignée du bras gauche. Les rameaux dépusés sur l'autel pouvaient aussi bien signifier une protestation contre la surdité d'un roi qui se refuse à entendre l'ordre des dieux, - comme 
c'est le cas dans la pièce d'Euripide, - ou un acte de confiance dans un roi qui fait sien le conseil de la divinité, - comme c'est le cas dans celle d'Eschyle.

$\mathrm{Au}$ surplus, on comprend bien pourquoi les feuillages restent sur la scène dans les Suppliantes d'Eschyle et sont emportés à la fin du prologue d'EEdipe. Le thème du respect aux suppliants domine tout le drame d'Eschyle et la verdure sacrée en est le symbole visible. Au contraire, la requête à $\mathrm{Edipe,} \mathrm{qui} \mathrm{sera} \mathrm{bientôt}$ oubliée en présence d'événements plus importants, sert simplement à montrer le respect presque religieux dont il est l'objet. Ironie tragique, ici encore. L'homme du monde qui s'est le plus et le plus cruellement trompé est celui dont on aura le plus dévotement honoré la clairvoyance. 